\title{
6 Future Trends (II): Mobile Devices for Education
}

\subsection{Introduction}

In the previous chapters of the book we have studied many useful resources to cover different levels of Bloom's taxonomy. Moreover, in Chapter 5 we started looking at the future with pedagogical agents that can bring advantages to education. In this chapter, we look at different devices and mobile applications for education. Figure 6.1 shows a general overview of this chapter.

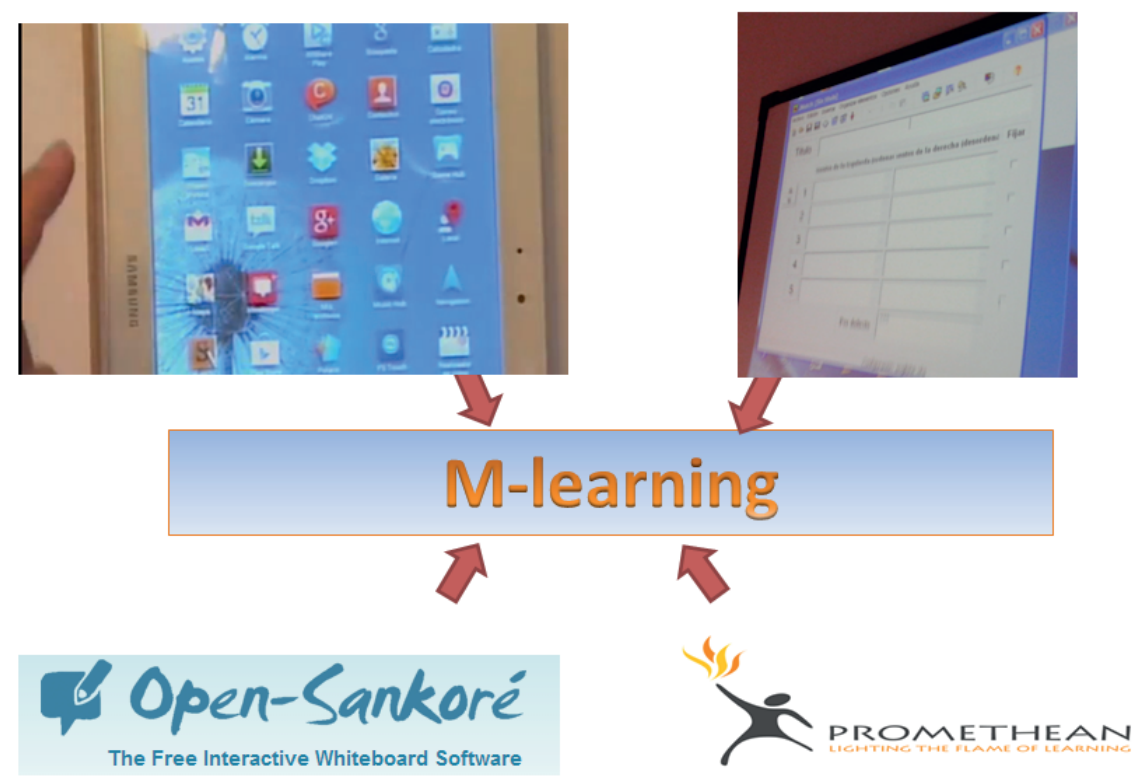

Figure 6.1: General overview of chapter 6

M-learning means the use of mobile devices for education. It is seen as the evolution of e-learning by some authors (Quinn, 2000; Pinkwart et al., 2003). M-learning is not a new term, and in some places, m-learning has been used for years, because it is easier in a class to have several tablets that students can easily move around, than moving all the students to the computer room. Moreover, digital whiteboards can allow teachers to give interactive lessons with the material they have created. Further, several students can interact with the board simultaneously, and the system will even save your annotations digitally, instead of just deleting them to make more space on the traditional blackboard! 
We cannot limit ourselves any longer to just using computers, we now have the opportunity to teach with new devices and software! And, since I know that new devices can bring new challenges, I would like to teach you in the first part of this chapter, the hardware. If you have never used a tablet before, Section 6.2.1 would be useful for you. Similarly, if you have never used a digital whiteboard, you may find interesting reading in Section 6.2.2.

However, tablets and digital whiteboards are just hardware. In fact, tablets, digital whiteboards, computers, hardware in general, these are all nothing without good software (and vice versa!). Therefore, in Section 6.3, I focus on the software that you can use to create educational activities with these devices, so that you can take advantage of your new knowledge and skills, and you can start using those devices to achieve pedagogical goals too!

\subsection{Hardware}

\subsubsection{Tablets}

Think about your computer for a minute. What have you seen when thinking about your computer? I suppose you have seen the screen, the keyboard, and the mouse. Now, think how these components can be combined into a thin device with a 7 to 10 inch screen. See Figure 6.2 for an example of a tablet.

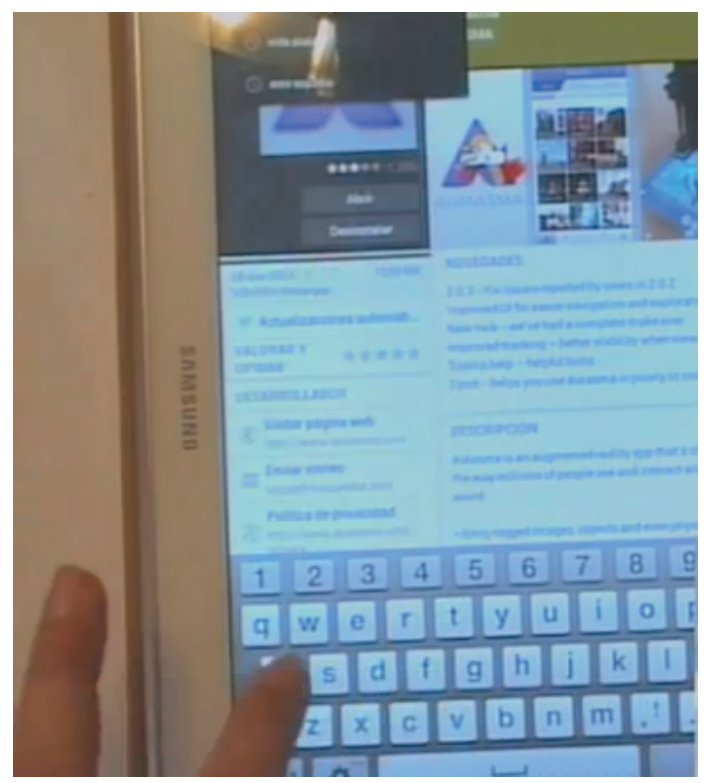

Figure 6.2: Example tablet 
Well, the screen is what you see and it has been integrated with the CPU (it is inside, trust me, you do not need to see the microprocessor!). The keyboard is also on-screen and there is no mouse! How is it possible to have no mouse? It is because the interaction is pretty much all the time with your fingers (sometimes, you can also use a pen if your model of tablet has it, but not all models use pens).

OK, I would like to buy a tablet, one of these like Figure 6.2, what are the features that I should look for? Well, several, namely: the screen, size, weight, operating system, processor, memory and hard disk capacity, battery, connectivity, audio, and possible accessories. Let's look at each of these features.

Regarding the screen, it is important to look at the type of screen: capacitive or resistive. Capacitive screens may cost more, but they are better because they are more responsive than resistive screens. However, my advice is that you try both types of screen so that you can choose which one feels best for you, but do not buy a resistive screen without trying a capacitive one. As you can imagine, given the importance of the screen in a tablet, there are many more things to check out, like the size: a screen of 6-7 inches can be a little small, but these are still good enough to read. However, for reading and working for longer periods with a tablet, a 10 inch screen is usually best.

The resolution of the screen and the type of crystal are also important factors to take into account when buying the tablet. A good, high definition, screen resolution should be able to reach $1024 \times 768$ pixels, and show high quality video fast. Videos are important, so try watching one before buying any tablet. This is also important because it is related to other factors like the processor and memory. It is possible that, even with a good resolution, you are not able to watch the video, since the processor is too slow or the tablet does not have enough memory. Finally, regarding the screen, you should ask if it has anti-glare technology. This is useful to avoid having problems, especially when trying to look at the screen from different angles.

Tablets are not usually much bigger than their screen, so the size of the tablet is usually determined by the size of the screen. Normally, a 7 inch tablet will weigh around 400 grams. This is another advantage of tablets, they are really light. In fact, in my case, I think that the cover of the tablet is heavier than the tablet itself. However, it does not mean you should forget about the cover. Given that tablets are pretty much their screen, it is fragile and if it breaks, you lose your tablet, so it is very important to put on a cover. In fact, two covers: one plastic, which is stuck onto the screen, and an outer cover to protect the screen from external knocks.

The operating system is also important because it will determine what software you can install, and how you are going to interact with the applications. Some people (in educational circles) are iPad enthusiasts because of the high quality software you can download. Other people prefer that the software they download is always free, and therefore prefer a free operating system such as Android. Good news, though! Now, you can choose! It usually depends on the policy of the school and your own ideas, so I leave that choice to you! 
The processor, memory and hard disk capacity are pretty similar to the features of a computer. All in all, a tablet is a computer too. So, it needs a processor that runs all the programs (e.g. 1.2 Ghz Tablet Processor). The higher the speed, the better the tablet will run the applications, and the faster it will respond to your requests (of course, the higher the price will be too!). As with computers, it is possible to have more than one processor. The hard disk size you'll need also depends on how you intend to use your tablet. For teachers, I have seen that in most cases, it is not necessary to have more than 8-16 Gb of internal memory, a $1 \mathrm{GHz}$ processor, and an $8 \mathrm{~Gb}$ hard drive (usually with tablets you upload many things to Dropbox or Google Drive, so you don't need to have a big hard drive, just enough for the operating system to work, and your apps). Nevertheless, before buying, always try the tablet, to see how it fits your needs, and ask if it is possible to extend some of its features later (e.g. with external cards or an external hard disk).

As is the case with your mobile phone, the battery is very important. You do not want to be looking for a plug every time you want to work with it. This would mean you lose the mobility advantage. Big, high resolution, color screens consume a lot of battery. So, many tablets not only have good batteries, but also good procedures to save energy, e.g. to turn the screen off when you are not looking at it. 8-10 hours of use is usually what you can get from one of these devices, if you are using it all the time. In that case, it is like a mobile phone, do not forget to charge it during the night. In some schools, they have charge stations, so that children leave the tablet there when the lessons finish, and next day they have their tablets ready for new lessons!

Connectivity is another important feature you cannot overlook. Nearly all tablets will have a WIFI connection so that you do not need a wire to get an Internet connection. However, WIFI is not the only way to get connected these days! You may want to connect to the Internet even when no WIFI is available, as is the case with your mobile phone. Therefore, you may need the tablet to have a $3 \mathrm{G}$ connection. If the tablet(s) is always going to be used in class, and the school has WIFI, then you do not need to pay more for the $3 \mathrm{G}$ connection. Otherwise, it could be a good feature to have. There is also the option of getting onto the Internet via USB, so you can ask for this possibility. Moreover, you should enquire about all the ports that the tablet has. Some models have mini-ports to connect to a computer so that you can share documents directly from the hard drive of the computer to the hard drive of the tablet (although you can always do that on-line with Dropbox or Google Drive). Other models may have a microHDMI port, which is very useful if you want to connect your tablet to a TV screen or a projector.

The quality of the audio is also important. Nearly all models have internal speakers so you do not need to bring external speakers. The idea is that tablets are easy and light to carry. It is also normal for them to have an input for earphones. So, as with other features, when you are watching a video to test the tablet, do not forget to evaluate the sound quality (stereo, Dolby, etc.). Finally, have a look at the accessories that the manufacturer of the tablet offers, and also at the tablet's compatibility with other devices. 


\subsubsection{Digital Whiteboards}

Think about your blackboard in class. What have you seen when thinking about your blackboard? I suppose you have seen some chalk, a duster, and the blackboard. Now, imagine that you do not need chalk and a duster any longer (so, you do not need to breathe chalk dust any longer, and get all your clothes dirty, particularly if they are black!) and that the blackboard is no longer static but you can interact with it, and not only you, but your students too. See Figure 6.3 for an example of a digital whiteboard (this is the digital whiteboard I use at my University). It is also possible to have smaller whiteboards that you can carry with you, these operate on batteries.

As was the case with tablets, there are many features to look into before choosing which digital whiteboard to buy. Normally, however, if you are not the Information and Technologies Communication coordinator, or a member of the computer staff, you will not be asked about the model of whiteboard to buy, so do not worry. Also, it is not normal to buy a digital whiteboard for your home. So, it is a much better idea to learn how to use the whiteboard that you find in class.

Digital whiteboards are becoming more common now in primary and secondary classes. So, let's take advantage of them! Are you interested? I hope so, because I want to give you a step-by-step guide to the use of a typical digital whiteboard.

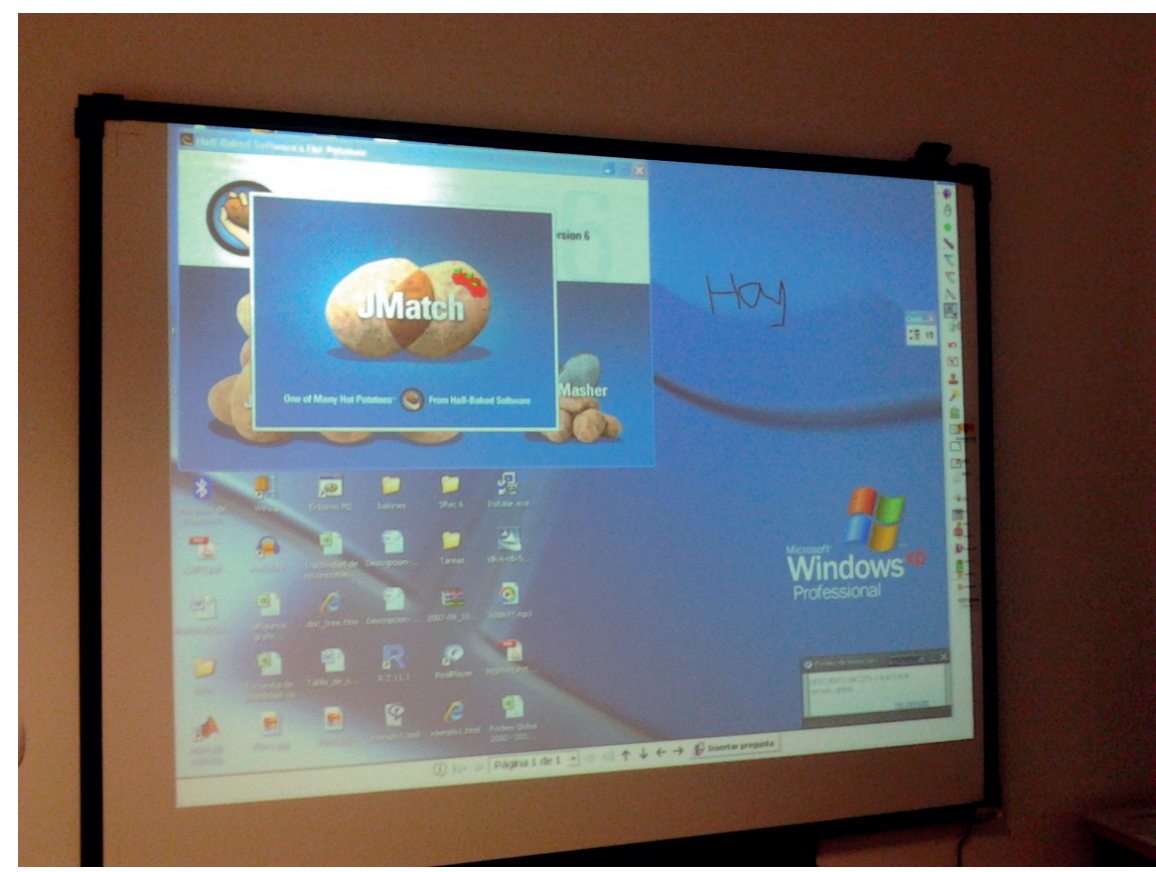

Figure 6.3: Example digital whiteboard 
First step. To turn the computer on. Yes, you have read correctly, I said turn the computer on. The digital whiteboard is connected to a computer and a projector, and you need the three things to work together. So, the first step is to turn the computer on (please, check that the computer is correctly connected to the digital whiteboard. Usually the people from the whiteboard company are responsible for installing everything correctly. If it's not connected, call them!).

Second step. To turn the projector on. You need the computer because it sends the information on its screen to the whiteboard. How can what is inside the computer be projected onto the digital whiteboard? Well, it uses the same technology that's used when you want a presentation from your computer to be shown on the wall, you use a projector. Again, as with the computer, the people who did the installation should have connected everything so that you just need to turn the projector on, and not check any wires. If there is a problem, you may need to call them or the school's computer staff. However, you should check that it isn't something really simple like that the projector is not plugged in, or it is telling you that you need to press a certain key because it is in stand-by mode. It is always a good idea to practice using the digital whiteboard several times on your own or with other teachers to get familiar with it before going to class.

Third step. To turn the digital whiteboard on. By now, you will have the computer with the operating system on, the projector on (although you do not see anything projected yet, we are close!) and you need to look for a button that is usually on the right of the digital whiteboard to turn it on. Have you noticed that a digital whiteboard seems a lot like a giant tablet? It could be a good idea to keep in mind, because it may help you using the whiteboard. Whiteboards are also usually responsive to your touch. It is also true that many digital whiteboards have pens, but the basic idea is the same: touch what you want to modify, open, close or have any interaction with! Do not worry, because, as is the case with digital tablets, you can undo nearly anything you do, and the digital whiteboard will not get broken. It is very important that you explore in order to learn more about the whiteboard's potential!

Fourth step. To run the digital whiteboard software. Although I will explain more about software for digital whiteboards in Section 6.3.2, I want you to know that, as I said before, with just the hardware you cannot do anything, you need to run some software. You can run it from the computer, now that you should be able to see everything projected on the whiteboard. Sometimes, it is also necessary to calibrate the whiteboard. This means that you touch, with pen or finger, on certain points of the whiteboard so that the whiteboard recognizes better the locations when you touch some part of it. If not, you may touch on one area of the screen, and the wrong program will be launched! 


\subsection{Software}

\subsubsection{For Tablets}

OK, so now you have a lot of information about the tablet's features. But, how can you use it? Well, there is something you already know, you interact with the tablet with your fingers. So, let's see a step-by-step example of how to use a tablet. First, you need to charge the battery. Remember, if it is the first time that you've charged it, it is usually a good idea to leave it charging all night. Do you already have enough battery to go through the example? Perfect! Let's start.

First step. To turn the tablet on. Unlike computers, tablets are usually on in stand-by power saving mode. This means that in many cases you just need to click on a button (on the right) to activate the tablet. See Figure 6.4 for an example ${ }^{39}$.

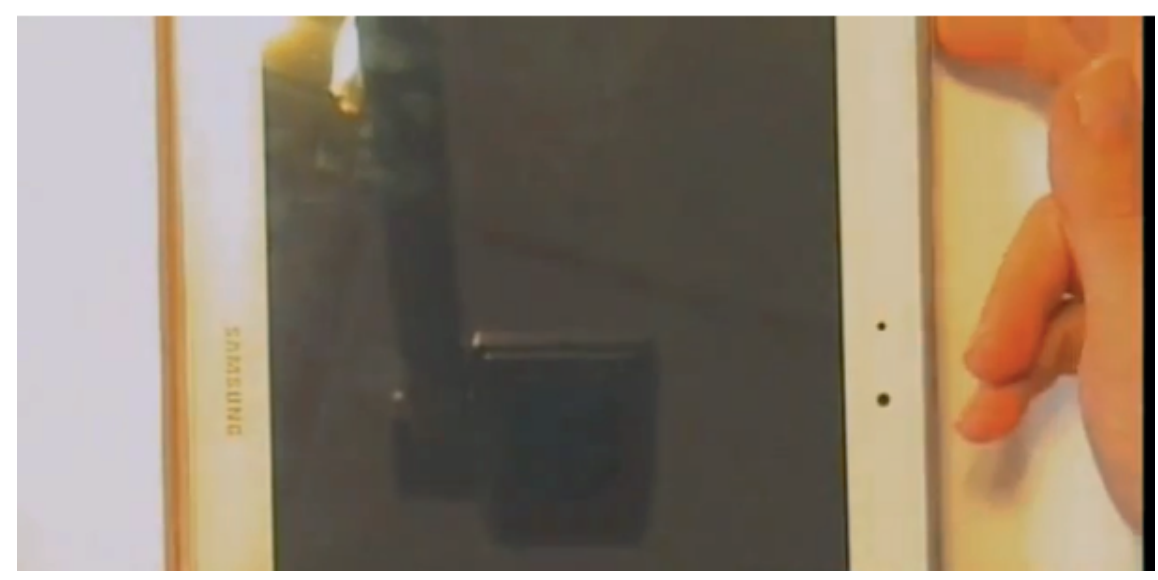

Figure 6.4: Turning on a tablet

If the tablet is off, then you may need more time to turn it on. You can see the logo of the maker of the tablet, the start of the operating system (like in a computer), and later the welcome screen. Take care! It is possible that the screen saver is on, so you will need to turn it off by touching the tablet screen with a left to right movement like the one shown in Figure 6.5.

39 All these images are from a video I recorded for my Spanish students, so if you know Spanish, you can watch the video at http://www.youtube.com/watch?v=5e9aBoopIkY 


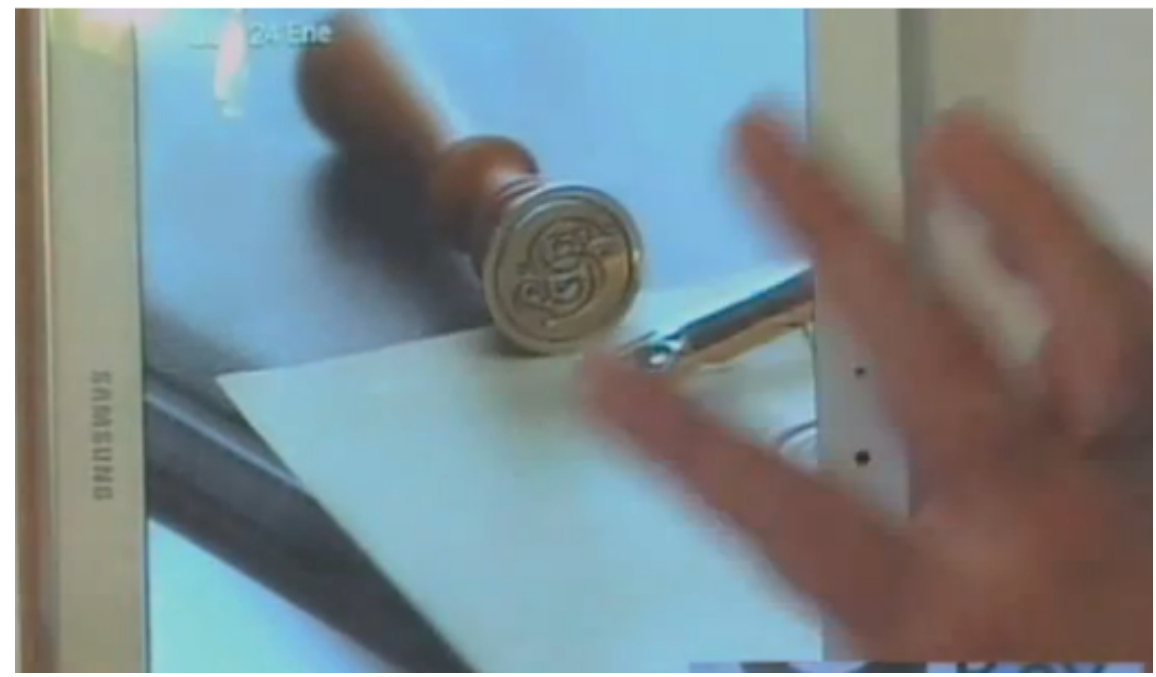

Figure 6.5: Turning on a sample tablet

Second step. To touch with your finger the app to use. In the case of tablets, the software programs are usually called apps, and they are saved directly to the screen. There are many screens and you can move your hand from left to right to see several screens containing all the apps. You can also organize your apps in folders so that they are classified according to certain rules. Anyway, you always touch the folder or app you want to open with your finger as you can see in Figure 6.6

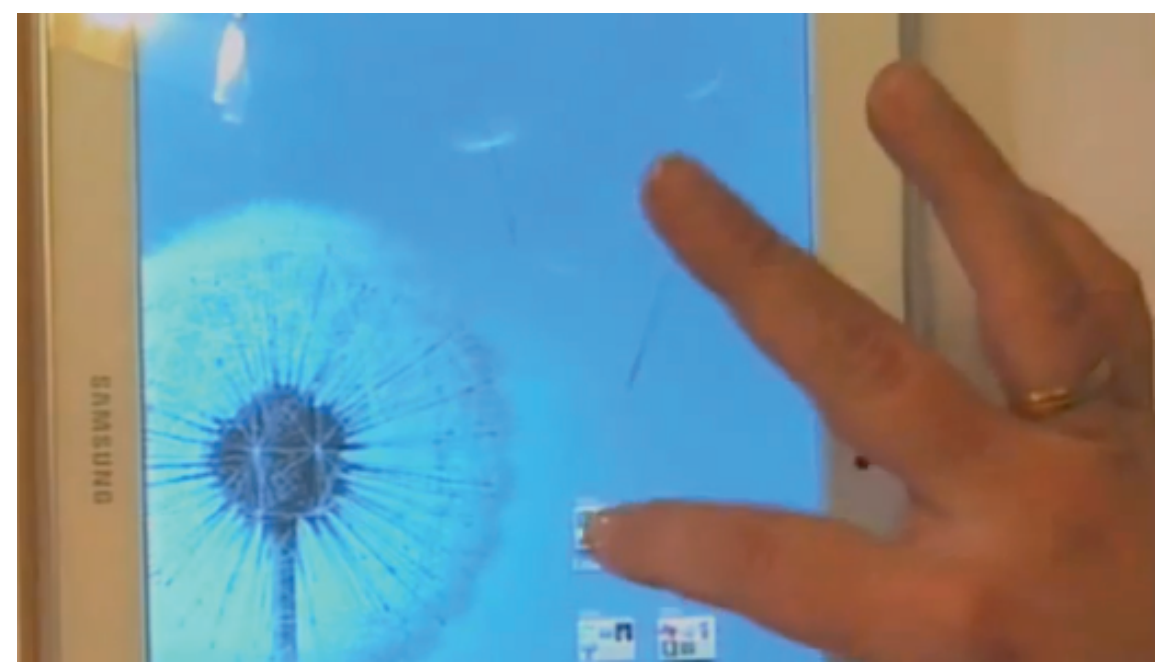

Figure 6.6: Opening a folder by touching it with the finger on the screen 
It is pretty intuitive. In general, anything you want to do with the tablet is just a matter of touching the screen in certain places. So, please explore all the possibilities, and if you get lost, and you do not know how to go back, do not worry, because tablets usually have a back button. If there is no back button, there is often another icon that may be different depending on the operating system (you can ask in forums) but there is always one button that gives you access to the main menu so that you can go back to the start and keep exploring!

Third step. To use the app. Apps usually cover the full screen when they are open. The reason is to facilitate your interaction with them. In my case, I have chosen to open an app to learn English that I love. It is called "English cards", and it is free. The idea is that you are presented with scenarios like the one shown in Figure 6.7 and the tablet tells you the name of an object (e.g. plant) so you have to look for that object in the scenario and touch it. It means that if you know what that object is, and you touch it right, the tablet tells you "Good!" and gives you another object name. Otherwise, the tablet tells you that it is wrong, so that you need to keep looking!

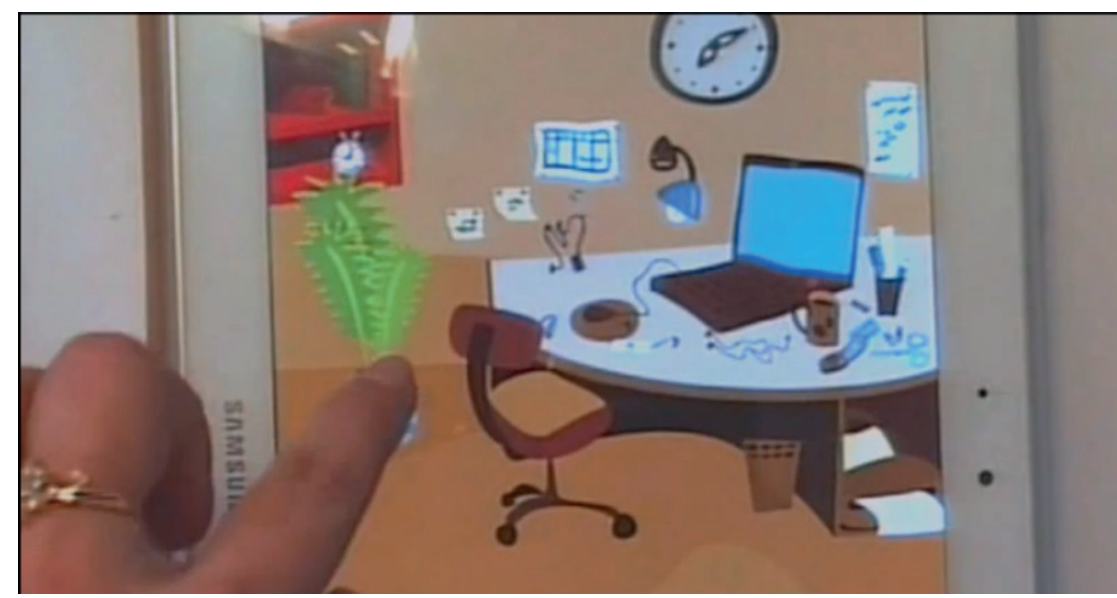

Figure 6.7: Interacting with an app in the tablet

Fourth step. To close the app and the tablet. Well, once you have finished using the app, you need to exit. It is a bit different from how you would exit a computer program. You just click on the back button of the tablet, or click on the options of the app as some of them have an option to log out of the app. It is also possible that if it is a free app you get some adverts. Finally, when you do not want to use the tablet any longer you can turn it off usually by clicking on the same button that you used when turning it on.

How can you find more interesting educational apps? Well, it depends on the operating system of your tablet. If it is Android, usually you will use "Google 
Play" 40 (see Figure 6.8) and if the tablet is an iPad you will use "Apple Store"41 (see Figure 6.9). The main difference is that for using Apple Store you need an iTunes account, and you type your password every time you want to download an app to the iPad. In the case of Google Play this is not necessary.

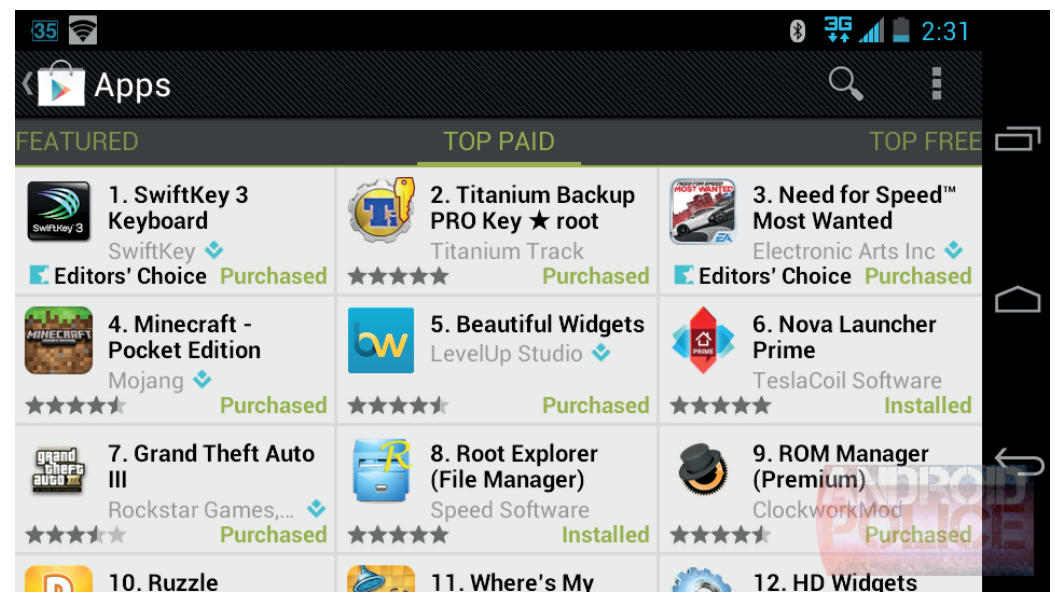

Figure 6.8: A screenshot of Google Play

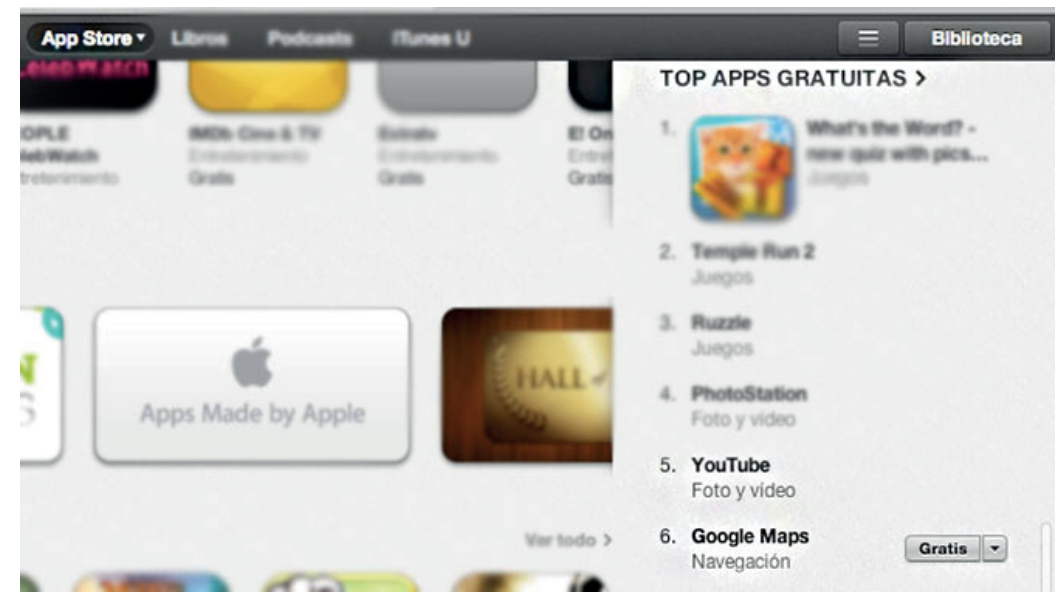

Figure 6.9: A screenshot of Apple Store

40 https://play.google.com/store/apps/category/EDUCATION?hl=en 41 http://www.apple.com/education/ipad/apps-books-and-more/ 
The rest is pretty similar. You touch with your finger the app you want to install (free or by paying), and select the "Install" option, and that's it! After a while, the tablet has installed the app on your desktop and you do not need to do anything more than try it. So, what's the difficult part? Choose which app to install. My advice is that you explore the possibilities and try them. There are many possibilities depending on the topic, the age of the children, the language and in general, the pedagogical goal that you want to pursue. As always, the technology is just a tool, and the creative mind is in the teacher!

\subsubsection{For Digital Whiteboards}

OK, so you already know that whenever you want to use the digital whiteboard, you need to turn on the computer, the projector and the digital whiteboard. With the hardware running, in order to take advantage of all the possibilities that the digital whiteboard can bring, we need to learn about the software you can use. There are many possibilities: Promethean activStudio ${ }^{42}$, SMART ${ }^{43}$, Interwrite ${ }^{44}$, etc. Usually, the software installed depends on the hardware. So, for instance, an Interwrite board (like the one that I have in my class) comes with Interwrite software. You can also use a free package like Open Sankoré ${ }^{45}$. The good news is that once you are familiar with one of these software packages, the rest are pretty similar. So, let's see a step-by-step example of how you can use, for instance, Interwrite.

First step. Once you check that the screen of the computer is projected on the whiteboard, you have to look for the Interwrite software icon on the desktop of the computer. If you do not see the desktop of your computer projected on the digital whiteboard, check if you have turned on the projector and the digital whiteboard. If you check both, and everything seems fine, call the technical staff. Figure 6.10 shows the image that usually appears as the icon for this software.

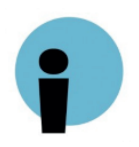

Figure 6.10: Logo of the example software (the background color may change to purple or green)

\footnotetext{
42 http://www.prometheanplanet.com/en/

43 http://smarttech.com/

$44 \mathrm{http}: / /$ www.einstruction.com

45 http://open-sankore.org/es
} 
Second step. To start using the software. Usually you can see a new menu bar on the right like the one shown in Figure 6.11. The icons tend to be intuitive. You can use different types of pens, just by clicking on the pen icon. There are many more possibilities, like using a white background, an image or anything you need. Moreover, everything you write on the whiteboard is saved. So, for instance, if you open a presentation and you make notes on the presentation, you can save these notes, and upload them to Dropbox so that students who could not attend the lesson can read them.

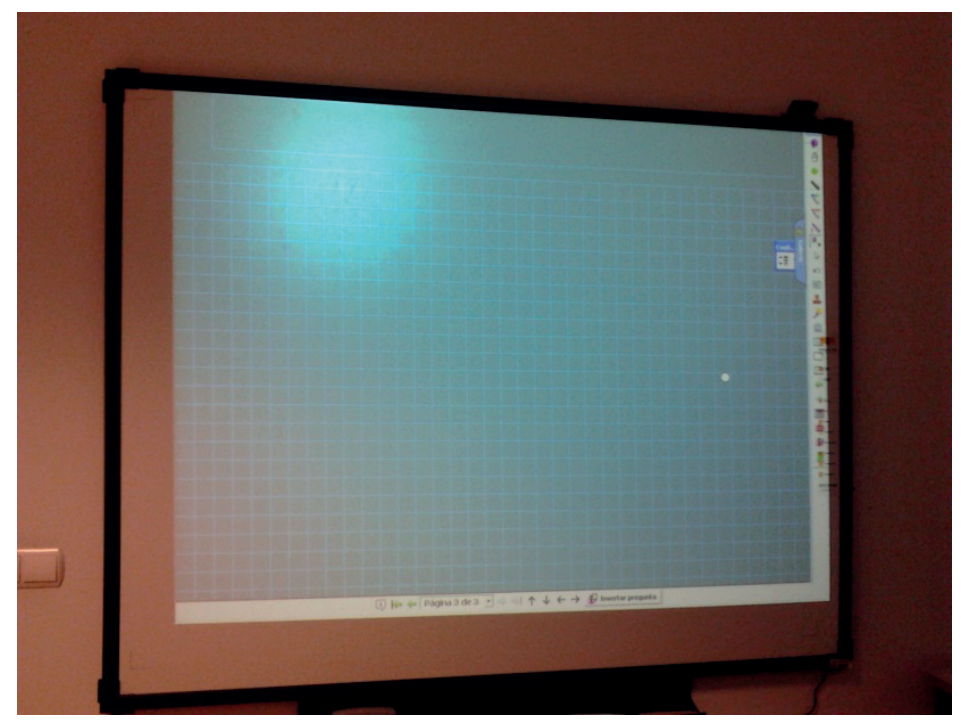

Figure 6.11: Example of notebook background to write with the pen on the digital whiteboard

Third step. To start using different software. You should not limit yourself to the software provided by the manufacturer of the digital whiteboard. There are many resources on-line waiting to be used for your students on your board. You can start with something that you are already familiar with. For instance, Hot Potatoes, do you remember Section 3.2? I suppose that you worked with Hot Potatoes with your computer, but now you have the possibility of projecting Hot Potatoes on the board for all your students to interact with it.

You just need to launch the program on the computer as shown in Figure 6.12. You can use it as if you were on a computer with the keyboard and the mouse (do not forget that you are using a computer too). However, I recommend that you try new things that are available for the whiteboard that you cannot use on the computer. For instance, Figure 6.13 shows the focus possibility. I love that option! You can put a black background to everything on the screen, except for a certain spot. 


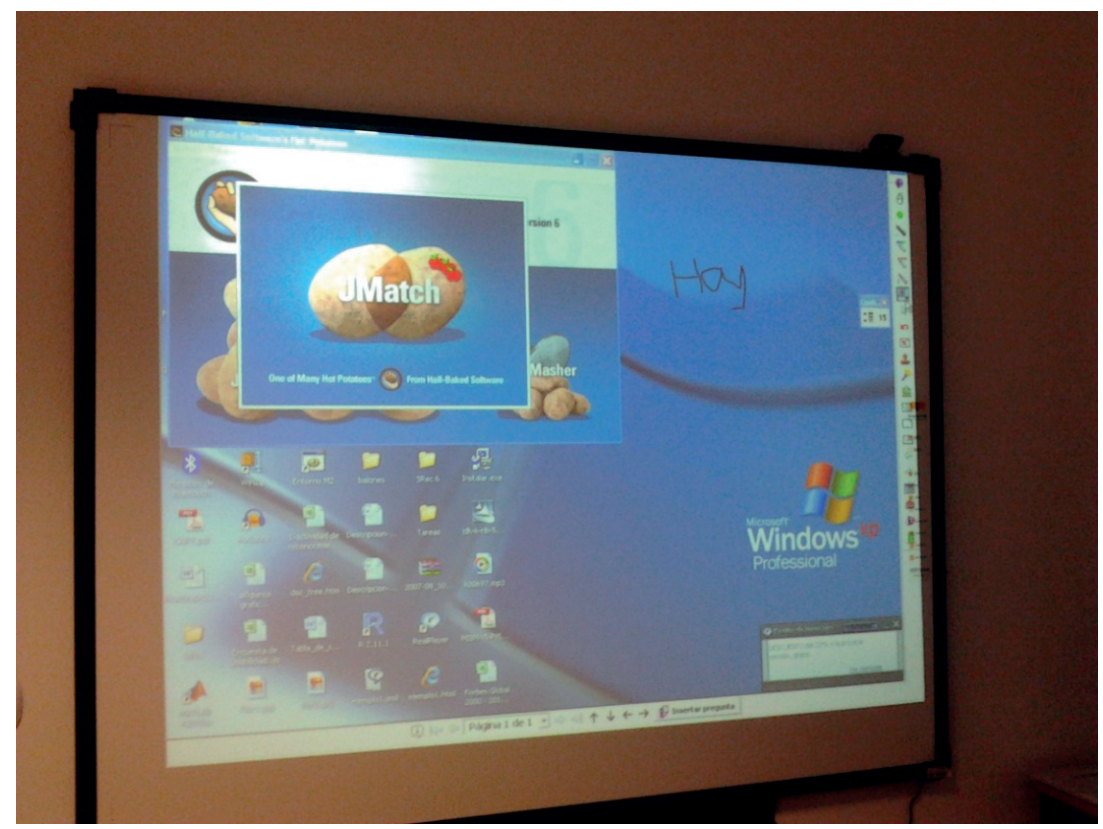

Figure 6.12: Using Hot Potatoes in the digital whiteboard

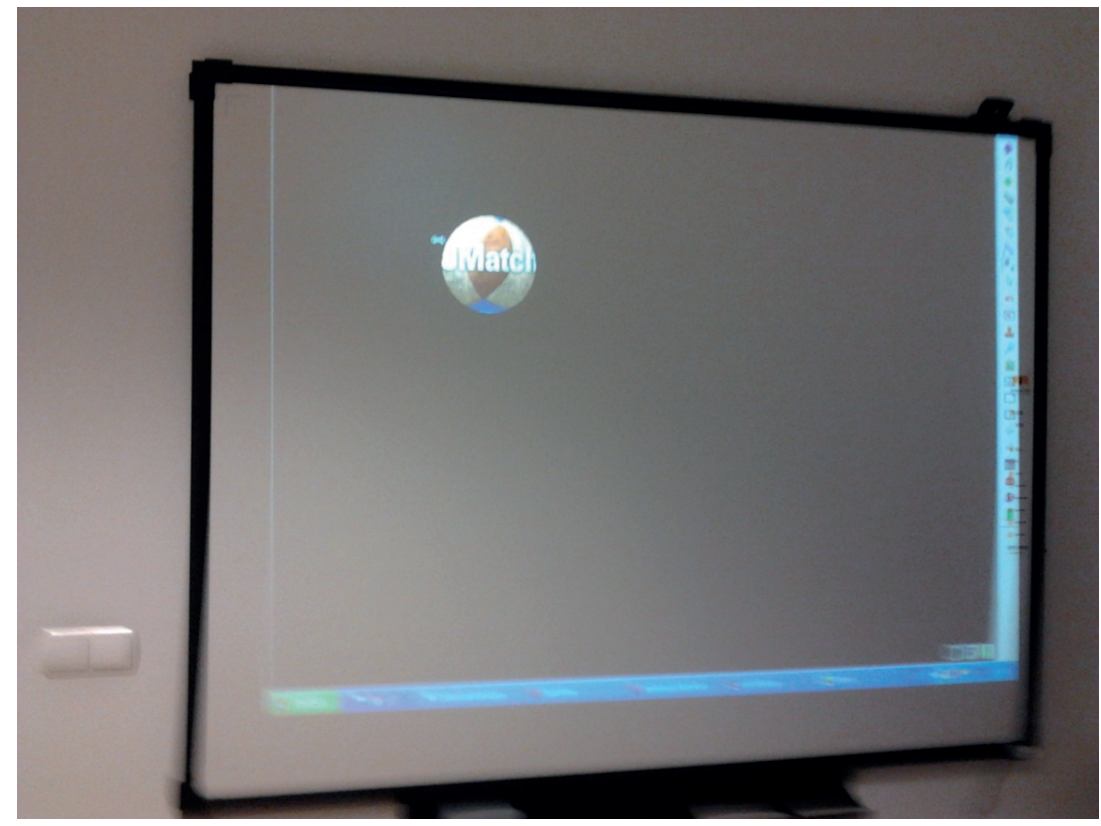

Figure 6.13: Using the focus feature of the digital whiteboard 
The spot can be very small at the beginning, and you can use that initial moment to start asking some questions of your students. For instance, if you want to do some matching, you ask them which Hot Potatoes program should we use? And when they say, JMatch, you can start making the spot bigger to focus on the right answer, and then move the focus to another part of the screen when you want to zoom in there.

There are many more possibilities, so please, explore, enjoy it, and do not forget to close the software. To do that, there is usually an exit option (in Interwrite it's a green door icon at the bottom). After closing the software, you need to turn off the computer, the projector, and the digital whiteboard, so that they are ready when you need to use them again. And, do not forget to bring some batteries if you are going to use the physical pen, as without batteries it will not work!

\subsection{Exercises}

Let's do several exercises to practice the content of this chapter. Remember that all exercises are solved at the end of the chapter, but do not read the solutions until you have tried to solve the exercises on your own first! It is also possible that you'll find different answers to these questions; in that case, you can tell us about them on the book's website.

1. Search on the Internet with your tablet. If you cannot use a tablet, find a video of someone searching on the Internet with a tablet.

2. Search for an educational application in Google Play if your tablet has Android, or Apple Store if it is an iPad. Install it on your tablet and practice with it. If you cannot use a tablet, find a video of someone using an educational application on the Internet.

3. Use the software on your digital whiteboard to make some notes about a presentation, save those notes and upload them to Dropbox. If you cannot use a digital whiteboard, find a video of someone making notes on a presentation using a digital whiteboard.

4. Open a JClic project in JClic player and project it on the digital whiteboard so that you can solve the exercises on the digital whiteboard. Later, ask your students to solve the exercises by interacting with the digital whiteboard, and observe their reactions (you can compare them to their reactions when using JClic on their computers). If you cannot use a digital whiteboard, find a video of a group of students solving some educational exercise on the board together. 


\subsection{Solved Exercises}

1. I like the video http://www.youtube.com/watch?v=nELQaWgdgQQ. In case it is no longer available when you try to watch it, just type the words: how to search on the Internet with a tablet, for instance, in YouTube, and you will see many interesting videos. Although, the most useful thing to do is to get access to a tablet, then you can follow the steps, touching the screen with your finger to control the browser (Google Chrome, Safari, etc.), and you can start typing in the search engine to find what you are looking for, or pasting the links that you want to see. You will soon see that it is pretty similar to searching on your mobile phone (but much better because the screen is bigger) or searching on your computer (and with the option of being outdoors and without any wire or heavy components to carry with you!).

2. I like the video http://www.youtube.com/watch?v=KtCfOMWVp14. In case it is no longer available when you try to watch it, just type the words: using an educational application in your tablet, for instance in YouTube, and you will see many interesting videos. Although, the most useful thing to do is to get access to a tablet, then you can follow the steps, touching the screen with your finger to search for the application, and touching the "install" button. So that you can touch the icon of the app installed in your tablet, and interact with it. The more you practice, the faster you will become familiar with this new type of interaction.

3. I like the video http://www.youtube.com/watch?v=75-7UqTy040. In case it is no longer available when you try to watch it, just type the words: using a digital whiteboard to make educational notes on a presentation, for instance, in YouTube, and you will see many interesting videos. Although, the most useful thing to do is get access to a digital whiteboard and then you can interact with it on your own. You will see how soon you become familiar with its powerful tools!

4. I like the video http://www.youtube.com/watch?v=RYuIh2IduDk. In case it is no longer available when you try to watch it, just type the words: children using a digital whiteboard in class, for instance, in YouTube, and you will see many interesting videos.

\subsection{References}

Pinkwart, N., Hoppe, H. U., Milrad, M. and Perez, J. (2003). Educational scenarios for the cooperative use of Personal Digital Assistant. Journal of Computer Assisted Learning, 19, 3, 383-391.

Quinn, C. (2000). M-Learning. Mobile, Wireless, In-Your-Pocket Learning. Linezine, Fall 2000. Found at: http://www. linezine. com/2.1/features/cqmmwiyp.htm. 